\title{
Spatio-temporal Analysis of Unstained Cells In-vitro
}

\author{
Nico Scherf ${ }^{1,2}$, Jens-Peer Kuska ${ }^{3}$, Ulf-Dietrich Braumann ${ }^{2}$, Katja Franke ${ }^{4}$, \\ Tilo Pompe ${ }^{4}$, Ingo Röder ${ }^{1}$ \\ ${ }^{1}$ Inst. f. Medizinische Informatik, Statistik u. Epidemiologie, Universität Leipzig \\ ${ }^{2}$ Translationszentrum für Regenerative Medizin, Universität Leipzig \\ ${ }^{3}$ Interdisziplinäres Zentrum für Bioinformatik, Universität Leipzig \\ ${ }^{4}$ Leibniz-Inst. f. Polymerforschung, Max-Bergmann-Zentr. f. Biomaterialien, Dresden \\ nscherf@imise.uni-leipzig.de
}

\begin{abstract}
The tracking of individual cells in time-lapse microscopy facilitates the assessment of certain characteristics of different cell types. Since manual tracking of an adequate number of cells over a considerable number of frames is tedious and sometimes not feasible, there is a vital interest in automated methods. We present a rather minimalistic approach for the tracking of unstained cells in cell culture assays. The proposed approach comprises background subtraction, an object detection method based on discrete geometrical feature analysis together with a validation of the resulting graph-structures. The main advantage of this approach lies in its computational efficiency.
\end{abstract}

\section{Introduction}

A proper way of understanding biological systems necessarily incorporates not only spatial organisation but also their dynamic development. Therefore, experiments assessing the spatio-temporal aspects of a biological system provide a promising way to unravel the underlying organisational principles. It is natural to extend the established methods of microscopy by taking a series of images in order to assess the temporal development. This so called time-lapse imaging will become a valuable tool in biological and medical research. The potential benefits are discussed in the review found in [1] and an overview of the technical aspects of time-lapse imaging and object tracking in a biomedical context can be found in [2].

A variety of different approaches to the analysis of time-lapse image data exists, where three main classes of approaches to object tracking can be distinguished according to [3]: (i) correspondence-based, (ii) transformation-based and (iii) contour-based methods. The latter two classes could also be subsumed into one. Correspondence-based methods base upon the detection of objects in a frame-by-frame manner. The correspondences between the detected objects are subsequently established. Transformation- or contour-based techniques focus on tracking the changes of initial objects over time. Here the contour-based aproaches became very popular over the last years $[4,3,5,6]$, especially in 
biomedical applications. Similar to [7], we decided to take the first approach of correspondence-based tracking since such methods are usually computationally more efficient. This is in particular true for cases when a great number of objects needs to be tracked.

We are interested in analysing the migration and proliferation of unstained cells in a cell-culture assay. The cells are not stained to rule out the effects of the staining agents on the behaviour of the cells. Usually phase-contrast microscopy is the common method of choice with the known drawbacks e.g. the typical halos and differences in the visual appearance of normal and mitotic cells [4]. Thus, we decided to use transmitted light microscopy and enhance the visibility of the cells by exploiting the effect, that slightly defocussed cells appear as rather bright objects (Fig. 1(a)).

The experiments are desgined to assess the behaviour of hematopoietic stemcells (HSCs) in vitro. The cells are cultured on a 3D silicone-substrate consisting of microcavities of different diameter, coated with certain molecules like fibronectin, heparin, collagen etc. to model the interactions between cells and extracellular matrix. The aim is to analyse the effects of these parameters (different proteins, spatial structure) on the behaviour of the cells. We are in particular interested in analysing the frequency and location of cell-divisions, different aspects of cell motility and also the structure of the obtained cellular genealogies (e.g. $[1,7])$.

\section{Materials and Methods}

The main problem of tracking unstained cells is the low signal-to-noise ratio. As can be seen from the image histograms, there is no clear distinction between the gray-level distribution of the cells and the background. This problem is further complicated by the visible structures of the substrate. As an example the gray-level profile along two different line is shown for a sample image in Fig 1(b). To alleviate this problem we subtract the background from every frame. The mean background image can be either obtained by taking a series of images

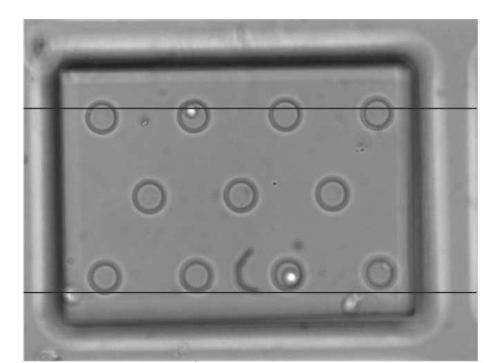

(a) Original image (gray-level profiles were taken at the the two lines)
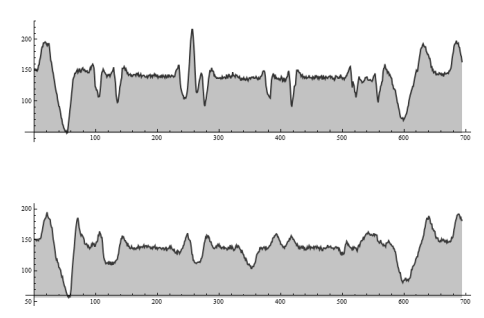

(b) Corresponding gray-level profiles

Fig. 1. Example of an image and its characteristic gray-level profiles 
before the cells are applied on the substrate or, if this is not possible, the mean intensity for every pixel must be estimated from the data. This simple approach to background estimation is computationally more efficient compared to more sophisticated methods (e.g. Gaussian mixture modelling, eigenbackgrounds etc.) and is sufficiently robust under the given image characteristics.

The pre-processed images are then segmented by thresholding and detection of connected foreground-components. Different geometrical features are calculated for the obtained objects to further remove falsely detected background structures. The most important features used here are the area, the largest and smallest object diameter as well as the discrete compactness and solidity of the objects. Deviations from typical values of compactness and solidity are used to detect objects that are likely to correspond to partially occluding or touching cells. Such objects are subsequently splitted into smaller structures. By taking the endpoints of a graph-based description of the objects [8] we obtain the center points to find a suitable splitting.

The results are depicted in Fig 2. The described method scales linearly with the number of foreground pixels. Thus, it is not as sensitive to the number of cells in the image as an approach based on active contours (e.g. [5]).

After object detection, the correspondence between consecutive frames needs to be established. To achieve this, we calculate the overlap of the objects in the previous and in the current frame. Ambiguities arise when an object overlaps with more than one object in the next frame. These cases are solved by detecting one-to-one mappings, removing the corresponding objects and then re-analysing the remaining objects. Such an approach critically depends on whether or not the sampling-theorem is met by image acquisition (for a detailed discussion see [2]). However, in some cases this requirement is not met and hence, errors in the cell correspondences can occur. In these cases we relax the requirements and allow correspondences between two objects that are a distance of not more than twice of the object diameter away. Furthermore, cells can be lost, in particular at the corners of the substrate structures. Thus, we need to keep track of such

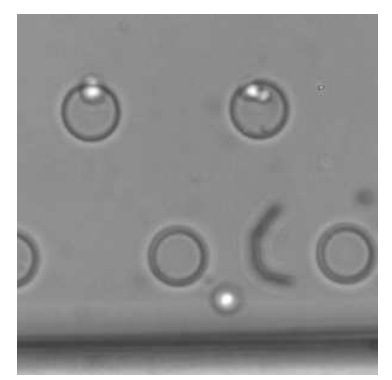

(a) Original image (zoom)

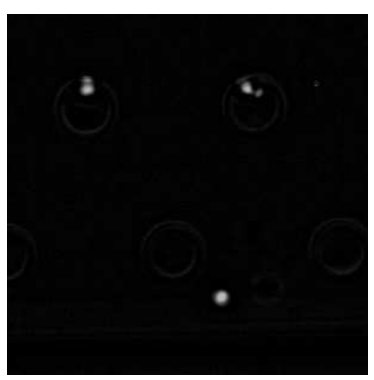

(b) Image with mean background removed

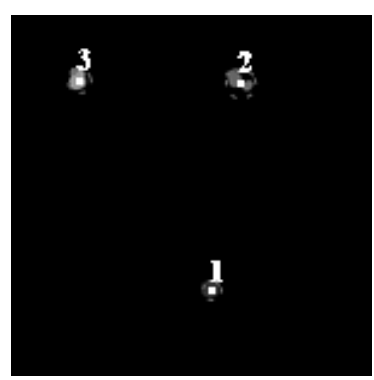

(c) Segmented objects

Fig. 2. Image preprocessing and segmentation 
lost cells. If a cell cannot be detected in the current frame, its position is stored and it is re-considered in the next frames for a certain period of time.

Sometimes cells overlap and consequently are falsely detected as a single object. If the fused object cannot be splitted as described above, the object is marked. When the cells eventually diverge, the correspondence is calculated between these newly seperated objects and the objects before the fusion.

\section{Results}

This work is still in progress, so presently only some preliminary, qualitative results are available to illustrate our propsed method. As an example an image sequence consisting of 4000 frames (examples shown here) taken every $60 \mathrm{sec}$ is analysed. Here we were in particular interested in the detection of cell divisions. Fig 3(b) shows the places, where cell divisions were detected. All of the mitotic events that occured during the observation time were successfully found. There was only one false positive detected cell division. It can be seen, that most of the cell divisions took place in the cavities or near the border structures of the substrate. The migrating behaviour of the cells was also analysed. In Fig 3(a) two examples of reconstructed cell tracks (over a period of 4000 frames) can be seen, showing different characteristics of migration patterns. In Fig 3(b) all places are marked, where a cell has been detected in the analysis. Here a tendency of the cells to move towards the upper left corner is apparent, which is most likely due to an incline of the petri-dish. And in Fig 3(c) a visualisation of reconstructed spatio-temporal cell-tracks is shown, where the track of one cell is marked in white showing both the migration pattern over time and the division events in a tree-like structure.

\section{Discussion}

We presented an approach to the problem of the spatio-temporal tracking of unstained cells in vitro. The results on the testing sequences are very promising

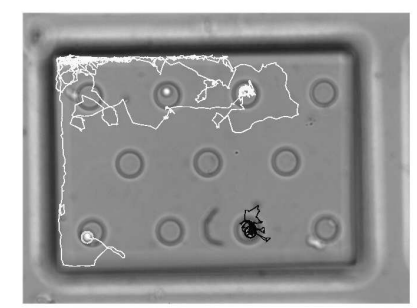

(a) Example tracks of two cells

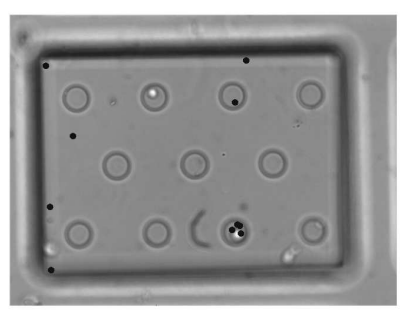

totic events (black spots)

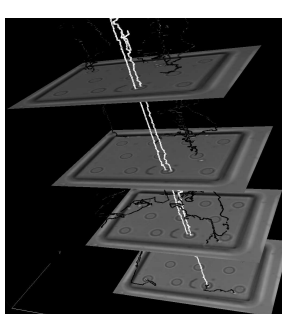

(c) Example of a reconstructed spatiotemporal trajectory (shown in white)

Fig. 3. Examples of information to be extracted from the time-lapse data 
and allow for an automated (or at least semi-automated) analysis of a variety of features of the spatio-temporal behaviour of the hematopoietic stem-cells in the presented culture-assays, which otherwise would not be feasible. The main advantage is that the presented approach is computationally attractive, thus allowing for an analysis in real-time. One important drawback is its sensitivity to segmentation errors and the resulting complications for object-linking. Some of the problems might be alleviated by a contour-based approach, which on the other hand would considerably increase the computational costs. As a next step, an objective validation of the method is needed as e.g. in [9] to evaluate the robustness of the method with respect to several parameters (e.g. cell density). It is to be expected that human interaction will be needed to correct for inevitably occuring errors during tracking, so a crucial point will be the optimisation of these interactions in order to obtain reliable results from the large amounts of data. Last but not least further measures for analysing the spatio-temporal trajectories need to be assessed.

\section{References}

1. Schroeder T. Imaging stem cell driven mammalian regeneration. Nature. 2008;453:345-351.

2. Meijering E, Smal I, Dzyubachyk O, et al. Microscopic Image Processing; 2008.

3. Yilmaz A, Li X, Shah M. Object contour tracking using level sets. Procs ACCV. 2004.

4. Li K, Miller E, Weiss L, et al. Online tracking of migrating and proliferating cells imaged with phase-contrast microscopy. Procs CVPRW. 2006; p. $65-72$.

5. Nath SK, Bunyak F, Palaniappan K. Robust tracking of migrating cells using fourcolor level set segmentation. Lect Notes Comput Sci. 2006;4179:920-932.

6. Padfield DR, Rittscher J, Sebastian T, et al. Spatio-temporal cell cycle analysis using 3D level set segmentation of unstained nuclei in line scan confocal fluorescence images. Procs ISBI. 2006; p. 1036-1039.

7. Al-Kofahi O, Radke RJ, Goderie SK, et al. Automated cell lineage construction: A rapid method to analyze clonal development established with murine neural progenitor cells. Cell Cycle. 2006;5(3):327-335.

8. Braumann U, Franke H, Hengstler J, et al. Graph-based quantification of astrocytes. Procs BVM. 2006; p. 379-383.

9. Godinez W, Lampe M, Wörz S, et al. Probabilistic tracking of virus particles in fluorescence microscopy image sequences. Procs BVM. 2008; p. 448-452. 